\title{
InFo Neurologie \& Psychiatrie punktet bei den Lesern
}

In der diesjährigen „Leseranalyse medizinischer Fachzeitschriften" (LA-MED) hat die InFo Neurologie \& Psychiatrie wie bereits im Jahr 2014 einen sehr guten vierten Platz belegt. Die Erhebung findet alle zwei Jahre unter niedergelassenen Ärzten in der Praxis sowie Chef- und Oberärzten in der Klinik statt und misst die Reichweite von Fachzeitschriften in der potenziellen Leserschaft. In zwei Wellen im Herbst 2015 sowie im Frühjahr 2016 waren 850 Fragebögen an repräsentativ ausgewählte Neurologen, Psychiater und Nervenärzte in Praxis und Klinik versandt worden. 329 Fragenbögen wurden zurückgeschickt, was einer Ausschöpfung von 40,5\% entspricht. Diese „Nettostichprobe“ kann laut LA-MED jedoch als „verkleinertes Abbild der Grundgesamtheit" gelten. Zur Grundgesamtheit werden in der Leseranalyse 13.332 Ärzte (7.252 Niedergelassene, 6.080 Chefund Oberärzte) gezählt. Erhoben wurden insgesamt 14 neurologisch-psychiatrische Fachzeitschriften sowie der Referenztitel „Deutsches Ärzteblatt“.1

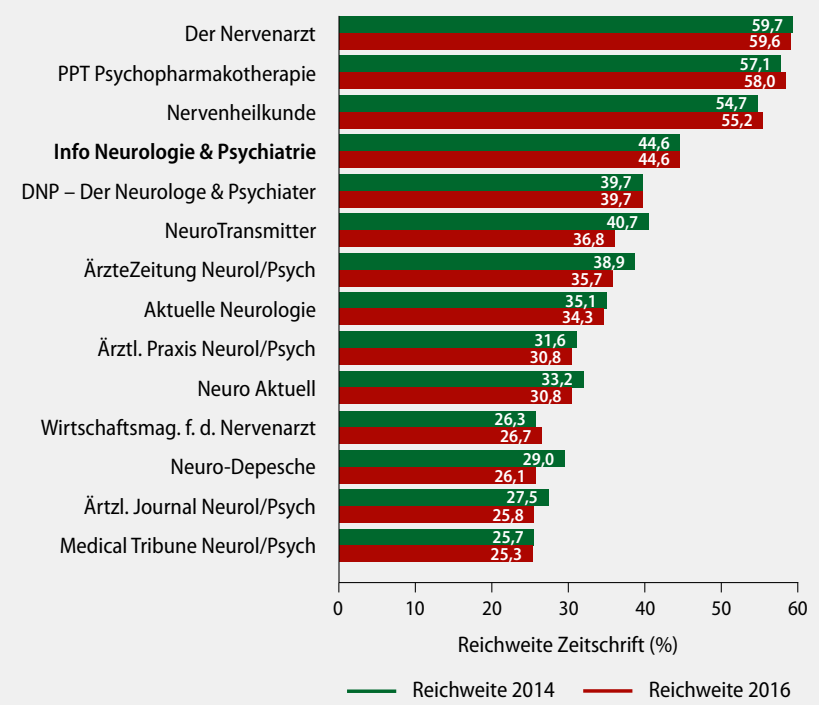

1 Entwicklung der Reichweite (Leser pro Ausgabe) in den Jahren 2014 und 2016 für verschiedene Fachzeitschriften (in Praxis und Klinik). (Anmerkung: Die Schwankungsbreite hängt von der Größe der Nettostichprobe sowie der ermittelten Reichweite ab und liegt für eine Sicherungswahrscheinlichkeit von $90 \%$ bei etwa $\pm 4-5 \%$.)

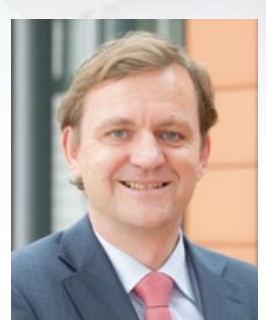

Professor Dr. med. Klaus Lieb, Mainz

Direktor der Klinik für Psychiatrie und Psychotherapie

Universitätsmedizin Mainz

Untere Zahlbacher Str. 8, 55131 Mainz

E-Mail: klaus.lieb@unimedizin-mainz.de

Die InFo Neurologie \& Psychiatrie erreichte unter Neurologen und Psychiatern in Praxis und Klinik eine Reichweite von $44,6 \%$, bei den Chef- und Oberärzten war die Reichweite mit $49,0 \%$ noch etwas höher. Diese Werte verdeutlichen den hohen Stellenwert unserer Zeitschrift in der Zielgruppe.

Fachzeitschriften $(67,9 \%)$ und Fachbücher $(68,0 \%)$ stellen für Neurologen, Psychiater und Nervenärzte in Praxis und Klinik nach wie vor die Informationsquellen dar, die sie beruflich „intensiv“ nutzen. Die Nutzung von medizinischen Fach-Webseiten nimmt jedoch langsam an Bedeutung zu, 32,0\% der Kolleginnen und Kollegen nutzen diese Dienste „intensiv“. Die Webseite www.springermedizin.de, auf der auch alle Inhalte der InFo Neurologie \& Psychiatrie zur Verfügung stehen, erreichte in der Befragung unter Neurologen, Psychiatern und Nervenärzten mit 49,2\% Reichweite mit Abstand den ersten Rang (Rang 2: www.aezteblatt.de $=40,6 \%$ ).

In der Grafik ist die Entwicklung der Reichweite (Leser pro Ausgabe) in den Jahren 2014 und 2016 für 14 verschiedene neurologisch-psychiatrische Fachzeitschriften dargestellt $(\triangleright$ Abb. 1). Auch wenn die Prozentwerte um etwa $\pm 4-5 \%$ ungenau sind, zeigt sich recht deutlich, dass die InFo Neurologie \& Psychiatrie unverändert eine vergleichsweise hohe Reichweite erreicht und von den Lesern sehr gut angenommen wird.

Unser Dank gilt an dieser Stelle daher einmal mehr unseren erstklassigen Autoren, die mit Zusammenfassungen und Kommentaren der wichtigsten Veröffentlichungen aus der internationalen Literatur sowie mit Beiträgen zur zertifizierten Fortbildung (CME) das wissenschaftliche Niveau unseres Fachblattes hochhalten. Ihnen als Leser wünschen wir unverändert viel Freude und einen guten Wissenszuwachs beim Studieren der Artikel. Für Kritik und Verbesserungsvorschläge sind wir jederzeit dankbar.

Klaus Lieb 